COMMENT. Clinical manifestations of cranial irradiation toxicity can occur in three stages: 1) acute increase in intracranial pressure related to brain edema; 2) subacute 'somnolence syndrome' developing after a 1 to 3 month interval and related to demyelination; and 3 ) chronic focal neurologic deficits, seizures, and cognitive, developmental, and endocrine deficiencies, caused by vascular damage and tissue necrosis and appearing months to years after radiation therapy. The delayedonset headaches reported in the above patients were vascular in type but were unassociated with demonstable vascular damage. The authors caution that cerebral angiography may exacerbate the migraine-like episodes.

Stroke as a late sequela of cranial irradiation for childhood brain tumors was reported in 11 children treated at the Southern California School of Medicine, Los Angeles (Mitchell WG et al. 1991). The addition of chemotherapy may potentiate damage to endothelial cells produced by irradiation causing a mineralizing microangiopathy. For a review of adverse effects of cranial irradiation and chemotherapy in the treatment of brain neoplasms, see Progress in Pediatric Neurology II, PNB Publishers, 1994, pp339-344).

\title{
BRAINSTEM TUMORS AND NEUROFIBROMATOSIS I
}

Seventeen children with neurofibromatosis type 1 (NF1) and MRI evidence of brainstem tumor are reported from the Children's Hospital of Philadelphia, PA. The mean age of diagnosis of NF1 was 55 months, and of brainstem tumor, 101 months. Headache was the most frequent complaint affecting 53\%, motor incoordination (41\%), cranial neuropathies (35\%), dysarthria (29\%), hemiparesis (12\%), papilledema (12\%), ataxia (12\%), head tilt $(12 \%)$, and seizures occurred in $6 \%$. The primary tumor site was in the medulla in $82 \%$, in contrast to the pontine location in non-NF1 patients with brainstem tumor. Shunt placement for hydrocephalus was required in $41 \%$, more frequent than in non-NF1 patients. Only $18 \%$ had clinical signs of progression. At a median follow-up of 52 months, 15 of 17 were alive. (Molloy PT et al. Brainstem tumors in patients with neurofibromatosis type 1: a distinct clinical entity. Neurology October 1995;45:1897-1902). (Reprints: Dr Patricia T Molloy, Division of Neurology, Children's Hospital of Philadelphia, 324 South 34th Street, Philadelphia, PA 19104).

COMMENT. Brainstem tumors in children with NF1 present with headache and cranial nerve palsies, they are often complicated by symptomatic hydrocephalus, are usually located in the medulla, and pathologically are benign or malignant astrocytomas, with variable outcome but usually prolonged survival. They can be distinguished from the typical NF-1 high-intensity foci on T2-weighted MRI (UBOs) by their mass effect and enhancement with gadolinium. Therapeutic intervention should be withheld pending evidence of progression, as determined by periodic neurologic examination and MRI. The authors estimated that $4 \%$ of their NF1 clinic population had a brainstem tumor. 